Research Article

\title{
Detection and Structural Characterization of Nucleophiles Trapped Reactive Metabolites of Limonin Using Liquid Chromatography-Mass Spectrometry
}

\author{
Yujie Deng, ${ }^{1}$ Yudong Fu, ${ }^{2}$ Shumin $\mathrm{Xu},{ }^{1}$ Ping Wang, ${ }^{1}$ Nailong Yang, \\ Chengqian Li, ${ }^{1}$ and Qing Yu ${ }^{1}{ }^{1}$ \\ ${ }^{1}$ Department of Endocrinology, The Affiliated Hospital of Qingdao University, 16 Jiangsu Road, Qingdao 266071, China \\ ${ }^{2}$ Department of Ophthalmology, The Affiliated Hospital of Qingdao University, 16 Jiangsu Road, Qingdao 266071, China \\ Correspondence should be addressed to Qing Yu; yuqing121985@126.com
}

Received 27 January 2018; Accepted 19 March 2018; Published 17 April 2018

Academic Editor: Federica Pellati

Copyright (c) 2018 Yujie Deng et al. This is an open access article distributed under the Creative Commons Attribution License, which permits unrestricted use, distribution, and reproduction in any medium, provided the original work is properly cited.

\begin{abstract}
Limonin (LIM), a furan-containing limonoid, is one of the most abundant components of Dictamnus dasycarpus Turcz. Recent studies demonstrated that LIM has great potential for inhibiting the activity of drug-metabolizing enzymes. However, the mechanisms of LIM-induced enzyme inactivation processes remain unexplored. The main objective of this study was to identify the reactive metabolites of LIM using liquid chromatography-mass spectrometry. Three nucleophiles, glutathione (GSH), $N$-acetyl cysteine (NAC), and $\mathrm{N}$-acetyl lysine (NAL), were used to trap the reactive metabolites of LIM in in vitro and in vivo models. Two different types of mass spectrometry, a hybrid quadrupole time-of-flight (Q-TOF) mass spectrometry and a LTQ velos Pro ion trap mass spectrometry, were employed to acquire structural information of nucleophile adducts of LIM. In total, six nucleophile adducts of LIM (M1-M6) with their isomers were identified; among them, M1 was a GSH and NAL conjugate of LIM, M2-M4 were glutathione adducts of LIM, M5 was a NAC and NAL conjugate of LIM, and M6 was a NAC adduct of LIM. Additionally, CYP3A4 was found to be the key enzyme responsible for the bioactivation of limonin. This metabolism study largely facilitates the understanding of mechanisms of limonin-induced enzyme inactivation processes.
\end{abstract}

\section{Introduction}

Dictamnus dasycarpus Turcz, known as Bai-Xian-Pi (BXP) in Chinese, belongs to the Rutaceae family. BXP has been widely used in Asian and European countries as an antipruritic, antidote, antibacterial, and anti-inflammatory agent. It is also used for the treatment of rubella, eczema, scabies, and jaundice. In addition, BXP displays diverse pharmacological properties, including antitumor, antiarrhythmic, antitinea, and smooth muscle-contraction activities [1]. Despite this, the safety of BXP has been questioned, and ingestion of BXP was reportedly associated with high incidence of liver injury. For instance, four cases of toxic hepatitis were reported in patients after taking a decoction made by boiling down the root of BXP [2]. In another clinical trial for the treatment of eczema, a standard mixture containing BXP was implicated in six of thirty-three cases of severe hepatitis [3].

Quinoline alkaloids, limonoids, sesquiterpenes, coumarins, flavonoids, and steroids have been explored as the major components of BXP [1, 4-8]. Limonoids have drawn much attention, and thus far a total of 25 limonoids have been isolated and characterized from BXP [1]; among them, Limonin (LIM) is one of the most abundant limonoids found in BXP [9]. Recent studies demonstrated that LIM has great potential for inhibiting the activity of drug-metabolizing enzymes and/or transporters such as CYP enzyme isomers and P-glycoprotein $[10,11]$. As far as we know, modulation of activity of drug-metabolizing enzymes and/or transporters will result in the alteration of the clearance of exogenous toxins and affects the hepatic detoxification functions. 
However, the mechanisms of LIM-induced enzyme inactivation processes remain unknown.

LIM is a furan-containing component. Many xenobiotics containing a furan unit are reported to be toxic and/or carcinogenic [12], such as furosemide [13], prazosin [14], teucrin A [15-17], 8-epidiosbulbin E [18-20], and diosbulbin B $[21,22]$. The toxic effects elicited by these furans are suggested to be attributed to their cis-enedial oxidative intermediate [12]. We hypothesized that LIM is metabolized to a cis-enedial intermediate (3, Scheme 1$)$, an electrophilic species, which may play a critical role in enzyme inactivation activities of LIM. In this study, we present the successful characterization of a cis-enedial intermediate of LIM and the identification of the cytochromes P450 (CYP450) enzymes responsible for the metabolic activation of LIM.

\section{Materials and Methods}

2.1. Chemicals and Materials. Mouse liver microsomes (MLMs), human liver microsomes (HLMs), recombinant human P450 enzymes, NADPH-regenerating system, glutathione (GSH), $N$-acetyl cysteine (NAC), and $N$-acetyl lysine (NAL) were purchased from BD Biosciences (Bedford, MA, USA). LIM (purity $>98 \%$ ) was obtained from Sigma-Aldrich (Sigma-Aldrich, St. Louis, MO, USA). Acetonitrile (ACN), methanol $(\mathrm{MeOH})$, and formic acid (FA) of LC/MS grade were obtained from Fisher Scientific (Pittsburgh, PA, USA). Water was purified with a Milli-Q system (Millipore, Bedford, USA) and was passed through a $0.22 \mu \mathrm{m}$ membrane filter before use.

2.2. Animal Studies and Sample Collection. Experiments with mice were carried out according to the guidelines for Animal Experimentation of Qingdao University (Qingdao, China), and the protocol was approved by the Animal Ethics Committee of the institution. Female Kunming mice $(20 \pm$ $5 \mathrm{~g}$ ) were obtained from the Experimental Animal Center of Qingdao University (Qingdao, China). Mice were housed 5 per cage and maintained in air-conditioned quarters with a room temperature of $20 \pm 2^{\circ} \mathrm{C}$, relative humidity of $50 \pm$ $10 \%$, and an alternating $12 \mathrm{~h} \mathrm{light/dark} \mathrm{cycle} \mathrm{and} \mathrm{allowed} \mathrm{to}$ acclimate for at least 1 week prior to the start of the experiment. Mice were fed with standard chaw diet and water and were allowed to eat and drink ad libitum. LIM dissolved in dimethylsulfoxide (DMSO) was orally administered to mice at a signal dose of $5 \mathrm{mg} / \mathrm{kg}$. Twenty-four-hour mouse urine and fecal samples were collected at room temperature by using metabolic cages. Blank urine and fecal samples were collected prior to the LIM treatment. These samples were stored under $-80^{\circ} \mathrm{C}$ before analysis.

2.3. Sample Preparation. One hundred and fifty microliters of ACN was added to $50 \mu \mathrm{L}$ of urine sample, then vortexed for $3 \mathrm{~min}$ and centrifuged at $16,100 \times \mathrm{g}$ for $10 \mathrm{~min}$ under $4^{\circ} \mathrm{C}$. The supernatant was concentrated to dryness under a gentle stream of nitrogen gas at $45^{\circ} \mathrm{C}$. The resulting residue was reconstituted with $200 \mu \mathrm{L}$ of $\mathrm{ACN} /$ water $(50 / 50, \mathrm{v} / \mathrm{v})$ containing $2 \%$ acetic acid, followed by centrifugation at
$16,100 \times \mathrm{g}$ for $10 \mathrm{~min}$ at $4^{\circ} \mathrm{C}$. Ten $\mu \mathrm{L}$ aliquot of the supernatant was injected into LC-MS/MS systems for analysis.

2.4. Microsomal Incubations. Liver microsomes with a final concentration at $0.5 \mathrm{mg} / \mathrm{mL}$ were incubated with LIM $(30 \mu \mathrm{M})$ for $60 \mathrm{~min}$ in the presence of GSH or NAC and NAL at a final concentration of $1.0 \mathrm{mM}$. The experimental incubation mixture consisted of $100 \mathrm{mM}$ potassium phosphate buffer ( $\mathrm{pH}$ 7.4), a prepared NADPH-regenerating system, and MLMs or HLMs. Stock solution of LIM was prepared in DMSO, and the final concentration of DMSO in the incubation did not exceed $1 \%(\mathrm{v} / \mathrm{v})$. After preincubated at $37^{\circ} \mathrm{C}$ for $15 \mathrm{~min}$ in a water bath, the reactions were initiated by the addition of LIM and were incubated at $37^{\circ} \mathrm{C}$ for another $60 \mathrm{~min}$. The reactions were terminated by the addition of an equal volume of ice-cold ACN containing 2\% acetic acid. The mixture was vortexed and centrifuged at $16,100 \times \mathrm{g}$ for $5 \mathrm{~min}$. Aliquots of supernatants were stored at $-20^{\circ} \mathrm{C}$ until analysis. Control incubations without NADPH-regenerating system, without substrate, or without liver microsomes were performed to ensure that the formation of the metabolites was microsome- and NADPH-dependent.

2.5. Chemical Synthesis. Five milligrams of LIM was completely dissolved in $400 \mu \mathrm{l}$ of acetone, then $50 \mu \mathrm{L}$ of saturated sodium bicarbonate solution, and $10 \mathrm{mg}$ of Oxone were added successively to the resulting solution. The mixture was stirred for $15 \mathrm{~min}$ at room temperature, followed by addition of $50 \mathrm{mg}$ of GSH or $22 \mathrm{mg}$ of NAC, both GSH and NAC were dissolved in $500 \mu \mathrm{L}$ of saturated sodium bicarbonate solution. The mixture was stirred for $30 \mathrm{~min}$ and then centrifuged; the supernatants were harvested and evaporated to dryness under a stream of nitrogen gas at $45^{\circ} \mathrm{C}$. The resulting residues were reconstituted with $500 \mu \mathrm{L}$ of $\mathrm{pH} 7.4$ PBS buffer, then $5 \mathrm{mg}$ of NAL was added and stirred for $30 \mathrm{~min}$ at $70^{\circ} \mathrm{C}$, the reaction was cooled to room temperature and filtered through a $0.22 \mu \mathrm{m}$ member filter, and then analyzed by LC-MS/MS.

2.6. Recombinant Human P450 Enzyme Phenotyping. To determine the specific P450 enzymes involved in the formation of reactive metabolites of LIM, a total of 10 human recombinant $\mathrm{P} 450$ s, including $\mathrm{P} 450$ s $1 \mathrm{~A} 2,2 \mathrm{~A} 6,2 \mathrm{~B} 6,2 \mathrm{C} 8$, 2C9, 2C19, 2D6, 2E1, 3A4, and 4A11 were screened. Conditions were equivalent to those of the microsomal incubations except that the microsomes were replaced by individual human recombinant P450 enzymes at a concentration of $25 \mathrm{pmol}$ enzyme with a total volume of $200 \mu \mathrm{L}$ in each incubation. Experiments were performed in triplicate.

2.7. LC-MS/MS Method. All samples were analyzed on a Thermo-Finnigan spectra system consisting of an Ultimate 3000 degasser, an Ultimate 3000 RS pump, an Ultimate 3000 RS column compartment, and an LTQ Velos Pro ion trap mass spectrometer (Thermo Scientific, San Jose, CA) coupled with an electrospray ionization (ESI) interface. The ESI interface was operated in a positive ion polarity mode. The 

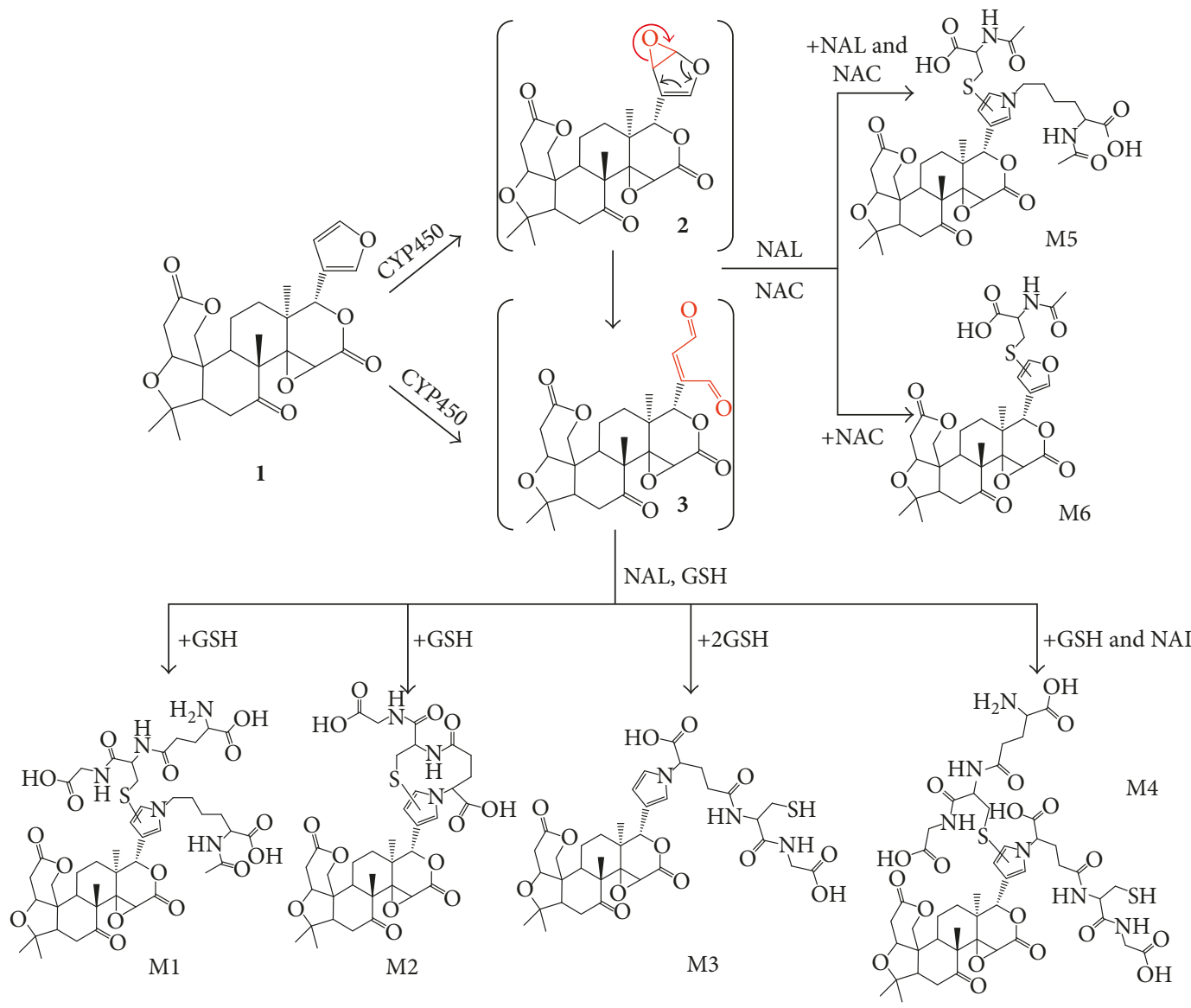

Scheme 1: Microsomal metabolism of the furan ring of LIM and the proposed pathway for the formation of LIM-derived conjugates.

voltage on the ESI interface was maintained at approximately $4.3 \mathrm{kV}$ and ESI capillary temperature was set at $300^{\circ} \mathrm{C}$. Nitrogen gas was used as the sheath gas and auxiliary gas which was set at 35 and 10 units, respectively. The collision energy was set at 35 with isolation width of $2 \mathrm{Da}$ for $\mathrm{MS}^{2}$. Chromatographic separation was performed on a Phenomenex Gemini C18 column $(5 \mu \mathrm{m}, 3.0 \mathrm{~mm}$ i.d. $\times 150 \mathrm{~mm}$; Torrance, CA, USA), and the column temperature was set at $35^{\circ} \mathrm{C}$. The mobile phase was $5 \%$ aqueous $\mathrm{MeOH}$ with $0.1 \%$ formic acid (mobile phase $\mathrm{A}$ ) and $95 \%$ aqueous $\mathrm{MeOH}$ with $0.1 \%$ formic acid (mobile phase B). The gradient was initiated at $90 \% \mathrm{~A}$ and held constant for $5 \mathrm{~min}$, followed by linear increases in B to $25 \%$ from 5 to $10 \mathrm{~min}$; to $60 \%$ from 10 to $30 \mathrm{~min}$, to $100 \%$ from 30 to $40 \mathrm{~min}$; and then held constant for $5 \mathrm{~min}$. The column was then reequilibrated with $90 \% \mathrm{~A}$ for $5 \mathrm{~min}$. The flow rate was set at $0.3 \mathrm{~mL} / \mathrm{min}$. The injection volume was $10 \mu \mathrm{L}$ for each sample. Data acquisition and analysis were performed using Xcalibur 2.2 version (Thermo Electron, San Jose, CA, USA).

Samples were also analyzed on a hybrid quadrupole time-of-flight (Q-TOF) mass spectrometer (Bruker micro Q-TOF, Bremen, Germany) with an electrospray ionization interface equipped with an Agilent 1200 series rapid resolution LC system. The mass spectrum data were acquired in the positive ion mode. The parameters of ESI-MS were set as follows: capillary voltage $(-4.3 \mathrm{kV})$, the nebulizer gas pressure $(1.2 \mathrm{bar})$, the dry gas flow rate $(8.0 \mathrm{~L} / \mathrm{min})$, and temperature $\left(220^{\circ} \mathrm{C}\right)$. The spectra were acquired at $2 \mathrm{~s}$ per spectrum in the range of $\mathrm{m} / z 100$ to 1200 . LC conditions were the same to those described above for the LTQ ion trap MS system. Data acquisition and analysis were performed using Bruker Daltonics data analysis 3.4 software.

\section{Results}

3.1. In Vitro Metabolic Activation of LIM. We proposed that the furan group of LIM played an important role in the LIMinduced inhibition of the activity of drug-metabolizing enzymes and/or transporters, and specifically this LIM is metabolized to the corresponding cis-enedial (Scheme 1, cisenedial 3), and the resulting electrophilic metabolites are responsible for the quench of many drug-metabolizing enzyme activities. LIM was incubated in MLMs or HLMs supplemented with GSH or NAC and NAL as trapping agents. The mixture was analyzed by a Thermo Scientific LTQ Velos ion trap mass spectrometer. Metabolites M1 and $\mathrm{M1}^{\prime}$ (retention times at 8.9 and $9.8 \mathrm{~min}$, resp.) were detected (Figures 1(b) and 1(c)) in both HLMs and MLMs incubations by scanning of an ion pair of $m / z 946 \rightarrow 817$, and $\mathrm{M} 1$ and $\mathrm{M} 1$ ' showed identical mass fragmental patterns with indicative characteristic secondary ion signals associated the cleavage of the GSH moiety (Figure 1(e)). The product ions at $m / z 928$ were derived from the loss of one water molecule $(-18 \mathrm{Da})$, and the product ions at $\mathrm{m} / z 871$ and 817 were 


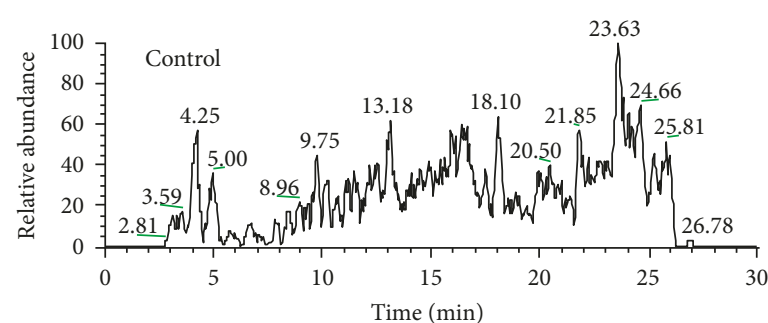

(a)

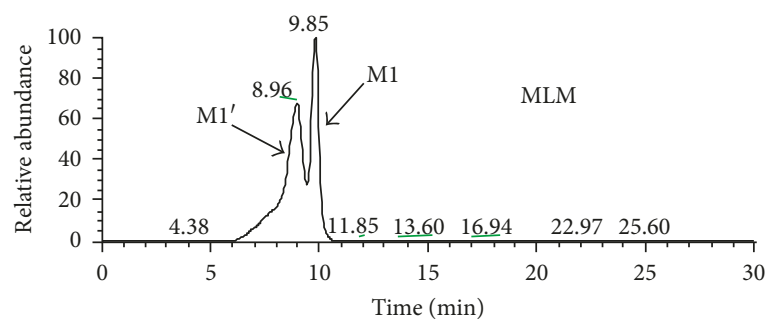

(c)

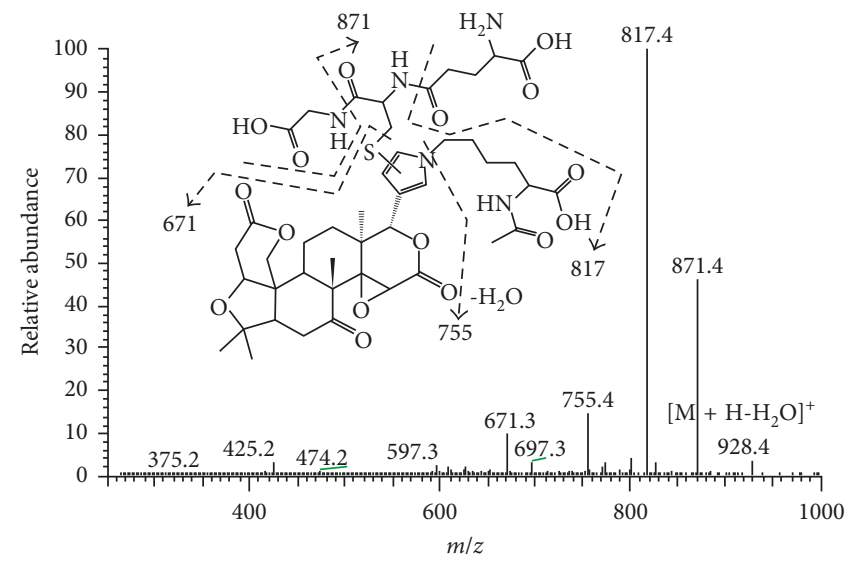

(e)

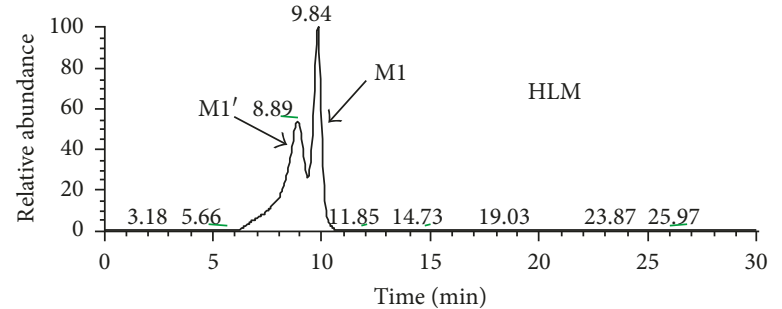

(b)

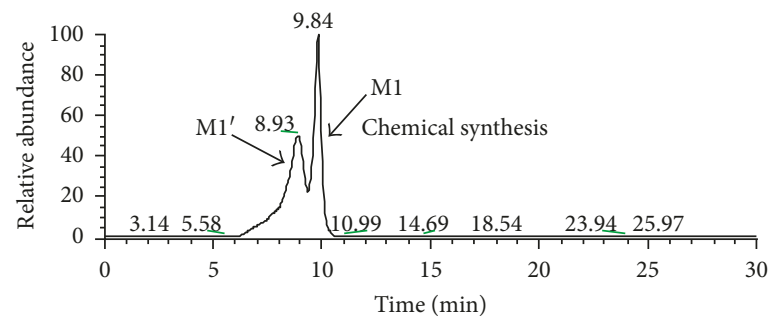

(d)

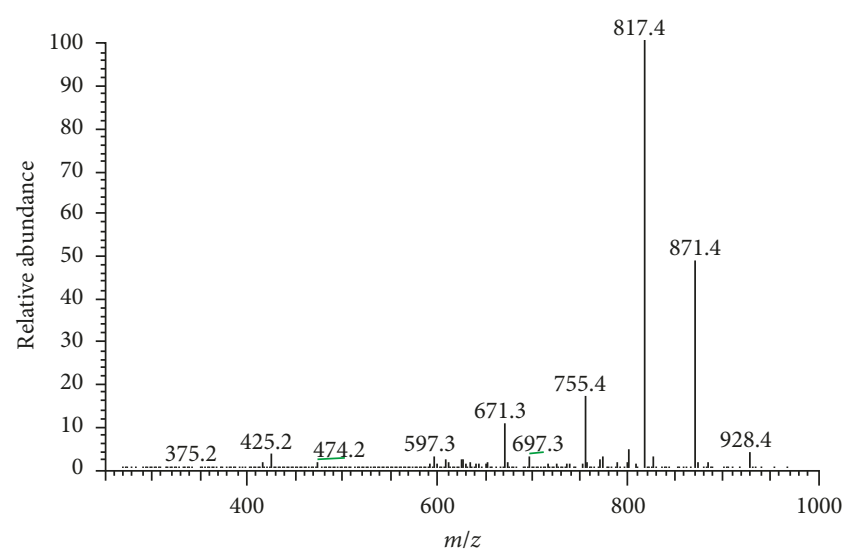

(f)

FIGURE 1: Extract ion $(\mathrm{m} / z 956 \rightarrow 817)$ chromatograms obtained from LC-LTQ MS analysis of microsomal incubations containing LIM, GSH, NAL, and NADPH in the absence microsomes (a), or in presence of HLMs (b) or MLMs (c). (d) Extracted ion $(\mathrm{m} / z$ 956 $\rightarrow 817) \mathrm{chromatogram}$ obtained from LC-LTQ MS analysis of synthetic M1 and M1'. (e) MS/MS spectrum of M1 generated in microsomal incubations (M1' showed the same MS/MS spectrum). (f) MS/MS spectrum of synthetic M1 (synthetic M1' showed the same MS/MS spectrum).

derived from the loss of glycine portion $(-75 \mathrm{Da})$ and $\gamma$-glutamyl portion $(-129 \mathrm{Da})$ from $\mathrm{m} / \mathrm{z} 946$, respectively. The mixture was also analyzed by LC/Q-TOF MS. M1 and $\mathrm{M1}^{\prime}$ showed their protonated molecule ion $[\mathrm{M}+\mathrm{H}]^{+}$at $\mathrm{m} / \mathrm{z}$ 946.4512 and $\mathrm{m} / \mathrm{z} 946.4513$ in positive ion mode, respectively; both of them matches the elemental composition of $\mathrm{C}_{44} \mathrm{H}_{60} \mathrm{~N}_{5} \mathrm{O}_{16} \mathrm{~S}$. No such conjugate was detected in the microsomal incubation system with the absence of NADPH (Figure 1(a)), indicating that the formation of M1 and M1' was mediated by the microsomal metabolism. To further characterize these two metabolites, we oxidized LIM with Oxone in acetone, followed by the addition of GSH and NAL; two major products formed in the reaction showed the same chromatographic and mass spectrometric identities (Figures 1(d) and 1(f)) as that of the products (M1 and M1') generated in the microsomal incubations. Unfortunately, we were unable to purify enough amount of the product for nuclear magnetic resonance (NMR) characterization.
Interestingly, mono-GSH adducts of LIM were also observed by selected reaction monitoring (SRM) scanning with ion transition of $\mathrm{m} / z 758 \rightarrow 683$ and $\mathrm{m} / z 760 \rightarrow 685$ in the positive mode. Under the transition of $\mathrm{m} / z 758 \rightarrow 683$, metabolites M2 and M2' (retention times at 12.9 and $12.2 \mathrm{~min}$, resp.) were detected in both MLMs and HLMs incubation systems (Supplementary Figures 1(B) and 1(C)). The tandem mass spectrometry (MS/MS) spectrum of M2 and $\mathrm{M}^{\prime}{ }^{\prime}$ were identical, with one of the major fragmental ions at $\mathrm{m} / \mathrm{z} 683$, indicating the loss of glycine $(-75 \mathrm{Da})$; the product ion at $\mathrm{m} / \mathrm{z} 740$ is postulated to arise from the elimination of $\mathrm{H}_{2} \mathrm{O}$ (Supplementary Figure $1(\mathrm{E})$ ). Further analysis by LC/Q-TOF MS demonstrated that M2 and M2' showed their protonated molecular ions at $\mathrm{m} / \mathrm{z} 758.2351$ and 758.2353, respectively, corresponding to the formula $\mathrm{C}_{36} \mathrm{H}_{44} \mathrm{~N}_{3} \mathrm{O}_{13} \mathrm{~S}$. On the basis of the observed mass spectrometric data, we propose that $\mathrm{M} 2$ and $\mathrm{M} 2$ ' are generated by intramolecular cyclization after GSH was conjugated to 


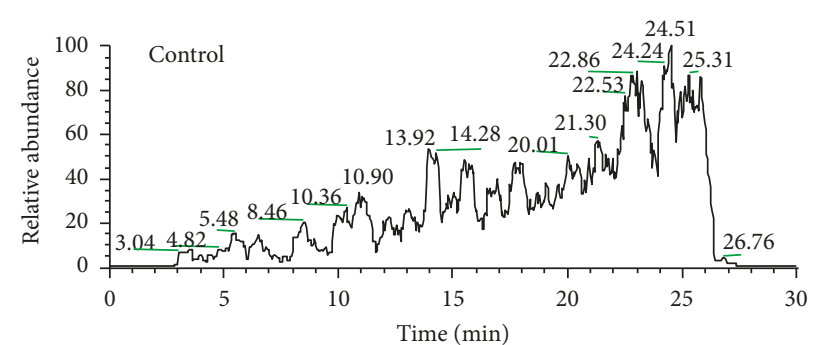

(a)

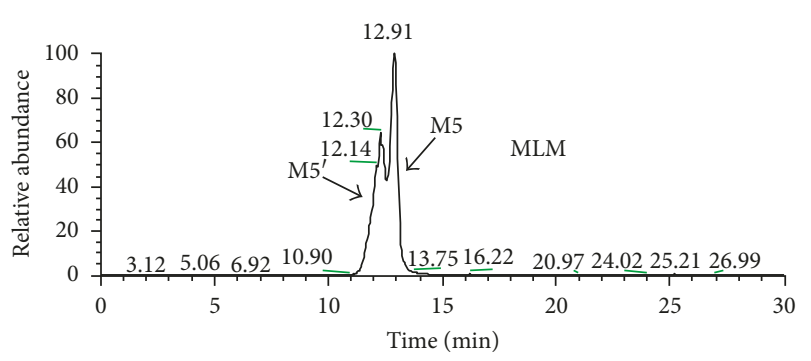

(c)

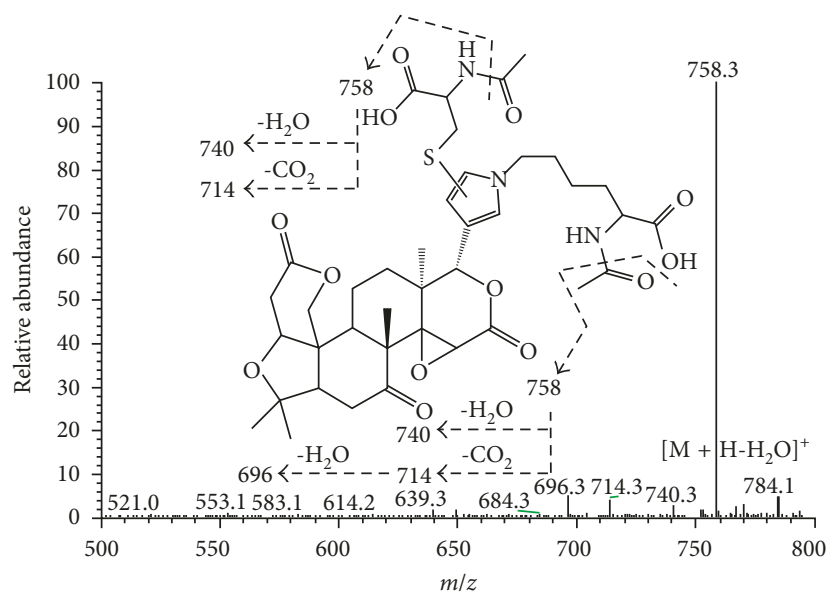

(e)

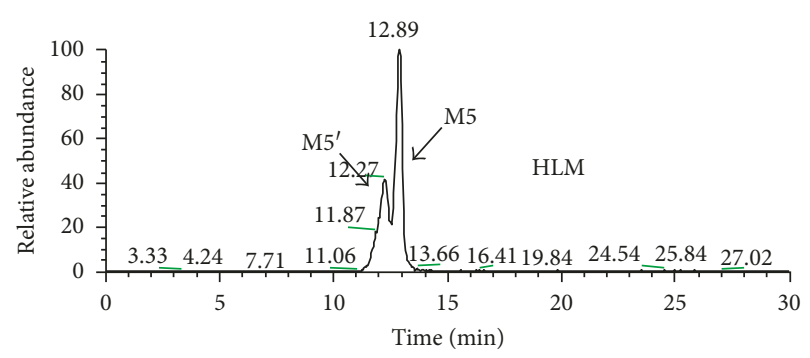

(b)

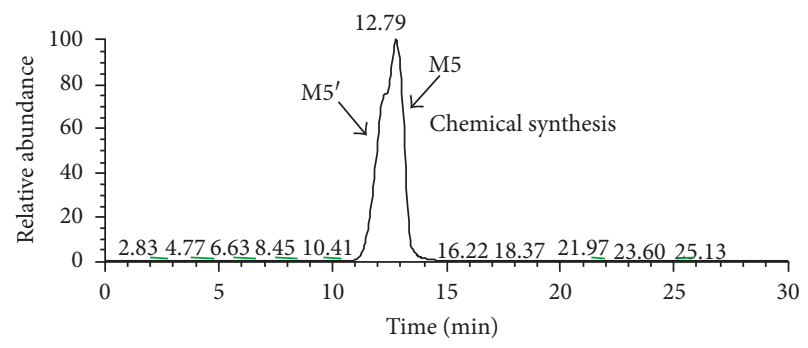

(d)

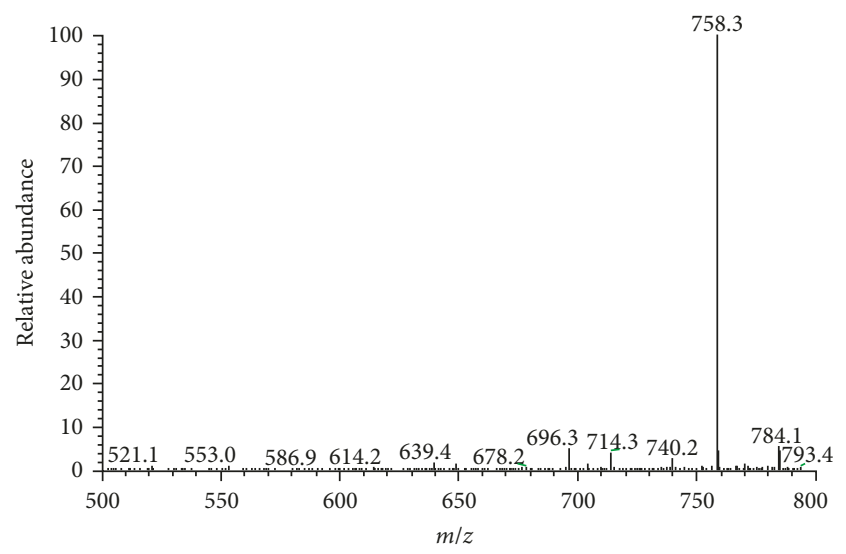

(f)

Figure 2: Extract ion $(\mathrm{m} / z 802 \rightarrow 758)$ chromatograms obtained from LC-LTQ MS analysis of microsomal incubations containing LIM, GSH, NAL, and NADPH in the absence microsomes (a) or in presence of HLMs (b) or MLMs (c). (d) Extracted ion ( $m / z$ 802 $\rightarrow 758$ ) chromatogram obtained from LC-LTQ MS analysis of synthetic M5 and M5'. (e) MS/MS spectrum of M5 generated in microsomal incubations (M5' showed the same MS/MS spectrum). (f) MS/MS spectrum of synthetic M1 (synthetic M1' showed the same MS/MS spectrum).

the cis-enedial intermediate which was derived from LIM. Under the transition of $m / z 760 \rightarrow 685$, four metabolites (M3, M3', M3", and $\mathrm{M}^{\prime \prime \prime}$ ) with retention times at 12.9, 12.3, 11.4 , and $10.6 \mathrm{~min}$, respectively, were detected in HLMs incubation system by mass spectrometry (Supplementary Figure 1(B)) and two metabolites (M3 and $\mathrm{M}^{\prime}$ ) with retention times of 12.9 and $12.3 \mathrm{~min}$ were observed in the MLMs incubation system; these metabolites showed identical mass fragmental patterns, provided major products ions at $m / z 742,685,657,614$, and 552 (Supplementary Figure $2(\mathrm{E}))$. These metabolites were further analyzed by LC/QTOF MS, and they showed their protonated molecular ion $[\mathrm{M}+\mathrm{H}]^{+}$at around $\mathrm{m} / z 760.2152$ in the positive ion mode, all of them in agreement with the elemental composition of $\mathrm{C}_{36} \mathrm{H}_{46} \mathrm{~N}_{3} \mathrm{O}_{13} \mathrm{~S}$. We propose that these metabolites were isomers of a pyrrole-GSH conjugate (Scheme 1). To further characterize the metabolites detected under the transition of $m / z 758 \rightarrow 683$ and $m / z 760 \rightarrow 685$, we analyzed the mixture of the biomimetic oxidation of LIM described above. As expected, M2, M2', M3, and M3' were all detected, based on their retention times, molecular ions, and MS/MS spectra (Supplementary Figures 1(D) and 1(E); Figures 2(d) and 2(e)).

Metabolites M4 and M4', with retention times at 9.6 and $8.5 \mathrm{~min}$, were observed by scanning of an ion pair of $\mathrm{m} / z 1065 \rightarrow 936$ in the positive ion mode (Supplementary Figure $3(\mathrm{~B})$ ). The MS/MS spectrum of M4 and M4' were identical which showed the major fragment ions associated with fragmentation of the GSH moiety (Supplementary Figure 3(E)). The product ions at $m / z 990$ and 936 were derived from the loss of glycinyl moiety $(-75 \mathrm{Da})$ and $\gamma$-glutamyl moiety $(-129 \mathrm{Da})$ from $\mathrm{m} / z 1065$, respectively. This indicates the participation of GSH in the formation of 


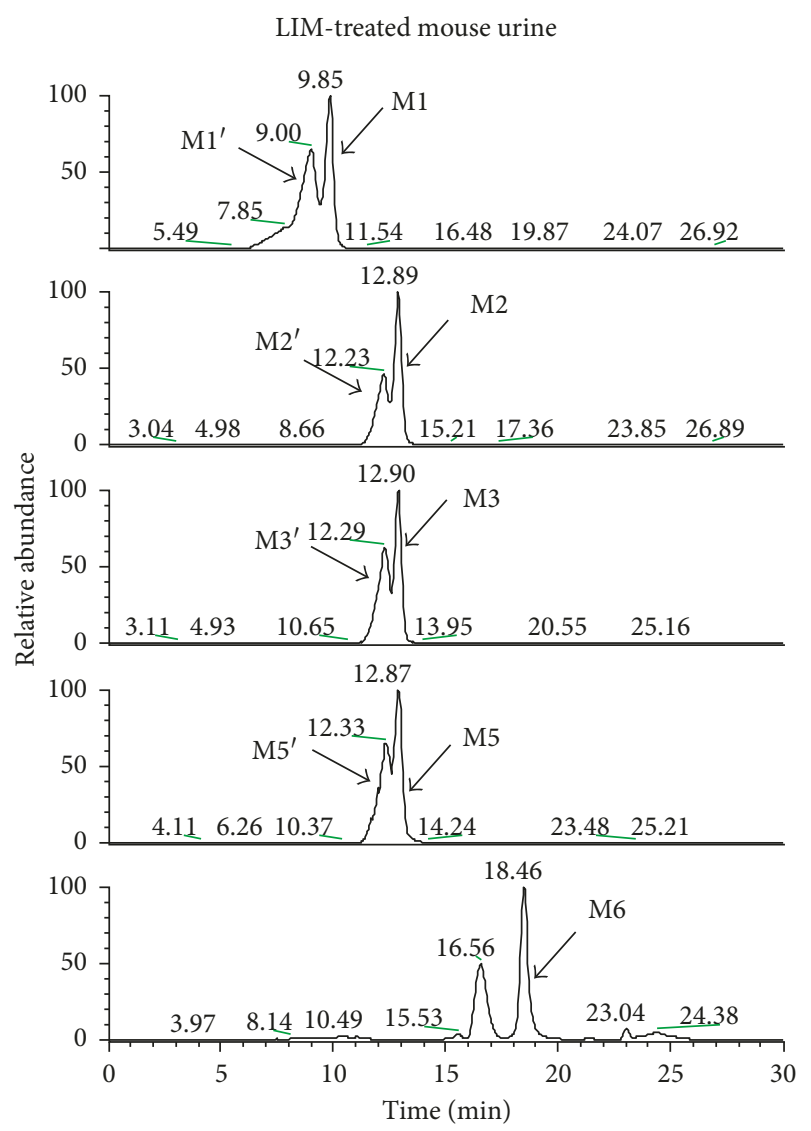

(a)

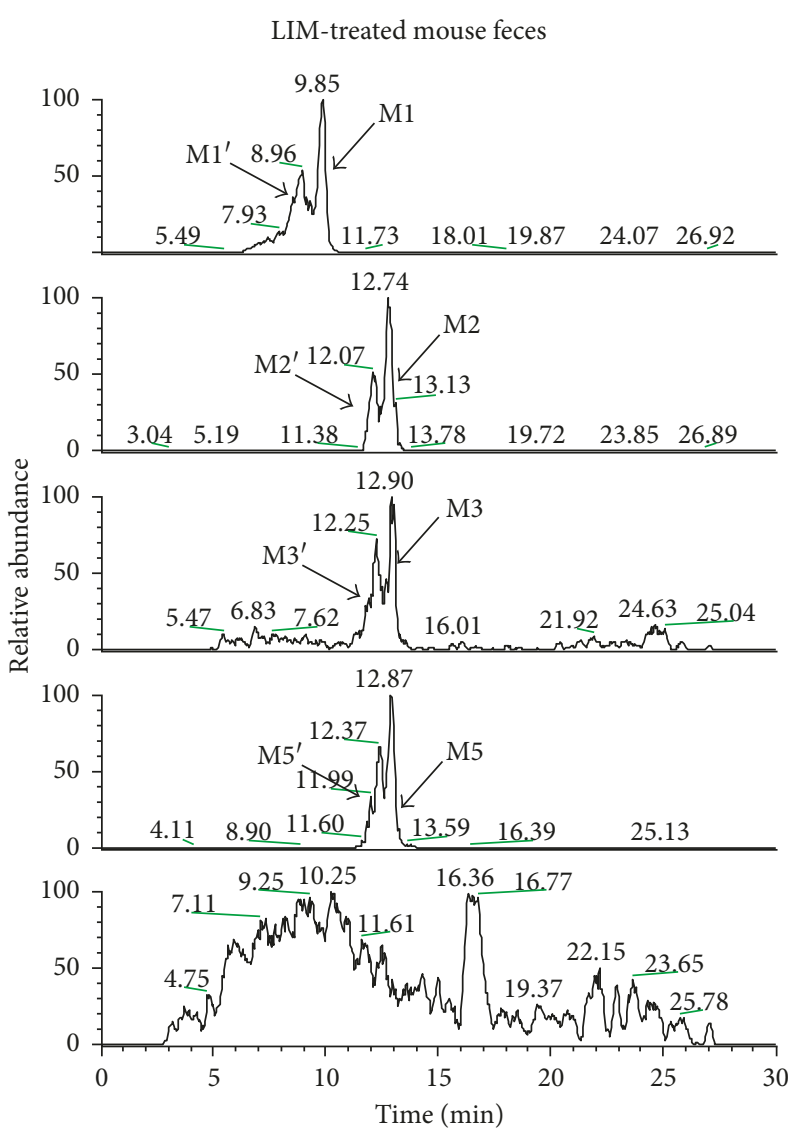

(b)

Figure 3: Extracted ion $(m / z 946 \rightarrow 817, m / z 758 \rightarrow 683, m / z 760 \rightarrow 685, m / z 802 \rightarrow 758$, and $m / z 632 \rightarrow 614$, represent M1, M2, M3, M5, and M6, resp.) chromatograms obtained from LC-LTQ MS analysis of urine (a), and feces (b) of mice after the treatment of LIM.

M4 and M4'. Further analysis by LC-Q/TOF MS showed its molecular ion at $\mathrm{m} / \mathrm{z}$ 1065.3431. The protonated molecular ions observed were consistent with the molecular mass corresponding to the elemental composition of $\mathrm{C}_{46} \mathrm{H}_{61} \mathrm{~N}_{6} \mathrm{O}_{19} \mathrm{~S}_{2}$, suggesting that $\mathrm{M} 4$ and $\mathrm{M}^{\prime}$ are derived from two molecules of GSH, which are LIM-derived di-GSH conjugates (Scheme 1).

In a parallel incubation, NAC in place of GSH was used to trap the LIM-derived cis-enedial intermediate. No such adducts like M1, M2, M3, and M4, which were found in GSH-fortified microsomal incubations, were observed in the NAC supplied microsomal incubation systems. Instead, we detected two metabolites (M5 and M6) most likely associated with NAC/NAL. Under the ion transition of $\mathrm{m} / z 802 \rightarrow$ 758, M5 and M5' were detected in both HLM and MLM incubation systems with retention times at 12.9 and $12.3 \mathrm{~min}$ (Figures 2(b) and 2(c)), respectively. These metabolites were further analyzed by LC/Q-TOF MS; M5 and M5' showed their protonated molecule ion $[\mathrm{M}+\mathrm{H}]^{+}$at $m / z 802.3217$ in positive ion mode, which matches the elemental composition of $\mathrm{C}_{39} \mathrm{H}_{52} \mathrm{~N}_{3} \mathrm{O}_{13} \mathrm{~S}$. The MS/MS spectrum of M5 and M5' identically showed the indicative characteristic neutral loss of one macular of water $(-18 \mathrm{Da})$ and successive losses of $44 \mathrm{Da}$ and/or $18 \mathrm{Da}$. The product ions at $\mathrm{m} / z \mathrm{784}, 758,740$, 714 , and 696 were assigned to $\left[\mathrm{M}+\mathrm{H}-\mathrm{H}_{2} \mathrm{O}\right]^{+},\left[\mathrm{M}+\mathrm{H}-\mathrm{CO}_{2}\right]^{+}$, $\left[\mathrm{M}+\mathrm{H}-\mathrm{CO}_{2}-\mathrm{H}_{2} \mathrm{O}\right]^{+},\left[\mathrm{M}+\mathrm{H}-2 \mathrm{CO}_{2}\right]^{+}$, and $\left[\mathrm{M}+\mathrm{H}-2 \mathrm{CO}_{2}-\mathrm{H}_{2} \mathrm{O}\right]^{+}$, respectively (Figure 2(e)). The formation of M5 and M5' were also found to be NADPH dependent (data not shown). To further characterize M5 and M5', LIM was oxidized with Oxone in acetone, follow by reaction with NAC and NAL. Two major products formed in the reaction showed the same chromatographic and mass spectrometric identities as that of the metabolites M5 and M5' generated in the microsomal incubation systems (Figures 2(d) and 2(f)). Besides M5 and M5', a mono-NAC adduct of LIM (M6) was observed under ion transition of $\mathrm{m} / \mathrm{z} 632 \rightarrow 614$ in positive ion mode. Fragmental ion generated from a natural loss of one macular of water $(-18 \mathrm{Da}), \mathrm{m} / \mathrm{z} 614$, was identified as the major fragmental ion; to further characterize this metabolite, we analyzed the mixture of the biomimetic oxidation of LIM described above. As expected, a product formed in the reaction showed the same chromatographic and mass spectrometric identities as that of the product (M6) generated in the microsomal incubations (Supplementary Figures 4(D) and $4(\mathrm{~F})$ ), and this metabolite was tentatively identified as mono-NAC adduct of LIM.

3.2. Metabolic Activation of LIM in Mice. To investigate the bioactivation of LIM in vivo, urine, and fecal samples collected from LIM treated mice were monitored by a designed 


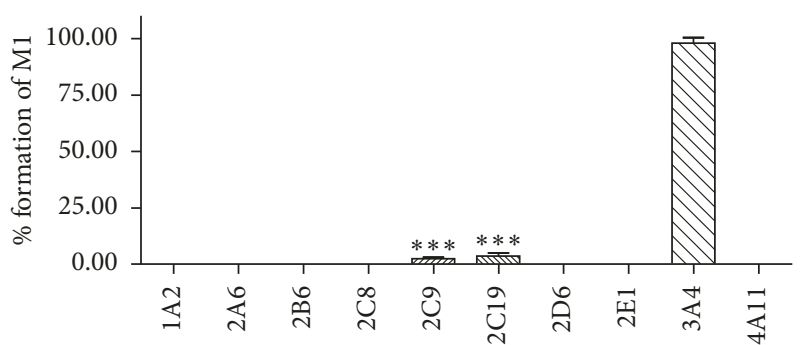

(a)

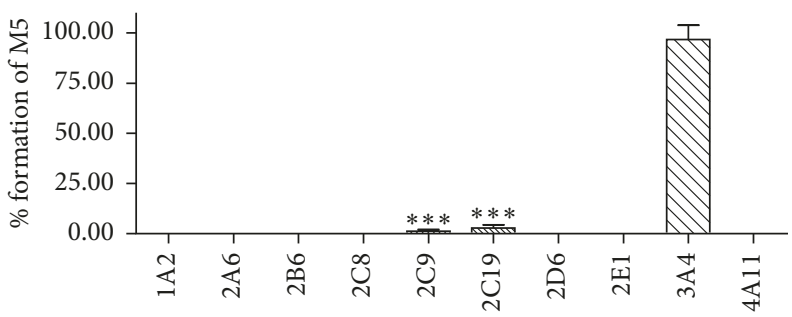

(b)

Figure 4: Rates of the M1 (A) and M5 (B) formation in incubations of LIM with recombinant human P450 enzymes.

selected reaction monitoring (SRM) template using LCMS/MS. One GSH and NAL adduct (M1), two monoGSH adducts (M2 and M3), one NAC and NAL adduct (M5), and one mono-NAC adduct (M6) were found in the urinary samples collected from the mice after given LIM (Figure 3(a)), except M6, these metabolites were also detected from the LIM-treated mouse fecal samples, while no such metabolites were observed from the blank mouse urine and fecal samples.

3.3. P450 Enzymes Responsible for the Bioactivation of LIM. In order to identify the P450 enzymes involved in the bioactivation, LIM was incubated with individual recombinant human P450s, including P450s 1A2, 2A6, 2B6, 2C8, 2C9, 2C19, 2D6, 2E1, 3A4, and 4A11, followed by monitoring the formation of M1 and M5. The bioactivation activities of each P450s were examined, and P450 2C9, 2C19, and 3A4 displayed the metabolic activity, with $3 \mathrm{~A} 4$ as the most potent one (Figures 4(a) and 4(b)). These results clearly confirmed that $\mathrm{P} 4502 \mathrm{C} 9,2 \mathrm{C} 19$, and $3 \mathrm{~A} 4$ were the primary enzymes that were involved in the bioactivation of LIM.

\section{Conclusions}

The citrus bitter principle limonin was first isolated in 1841, but intensive investigation of its bioactivity did not commence until the last decade. Metabolism study of LIM is still very limited, possibly due to little attention being paid to its toxic effects. The unexpected inhibition activity of LIM towards the drug-metabolizing enzymes and/or transporters led us to investigate the metabolic activation of LIM. We hypothesized that LIM is metabolized to an electrophilic species, a cis-enedial intermediate, then reactive with the nucleophilic side residues of proteins (thiol, alcohol, phenol, carboxyl, and amine), and led to the inactivation of protein functions.

In our current study, GSH which contains two nucleophilic functional groups, including a sulfhydryl (the cysteine residue) and an amino (the glycine residue) group, NAC and NAL each contains a nucleophilic amino group in its side chain, were chosen as the trapping reagents to trap the potential LIM-derived cis-enedial intermediate in mouse and human liver microsomal incubation systems after exposure to LIM. LC-MS/MS analysis showed a total of six LIM-derived conjugates (M1-M6) with their isomers in the microsomal incubations. On the basis of the molecular ions obtained from high-resolution mass spectrometry, six LIMderived pyrrole derivatives are postulated to be formed in the microsomal incubation systems. Mechanistically, a sulfhydryl group and a primary amine group participate in the formation of the pyrrole derivatives. The pyrrole production possibly starts with the reaction of the cis-enedial intermediate with the sulfhydryl residue of GSH or NAC by Michael addition to form the corresponding GSH or NAC conjugate, which subsequently reacts with the amino residue of NAL to form a Schiffs base followed by intramolecular cyclization and dehydration to produce the pyrrole derivative. For the formation of $\mathrm{M} 1$, the sulfhydryl and amino groups came from GSH and NAL, respectively. For the generation of $\mathrm{M} 2$ and $\mathrm{M} 3$, the nucleophilic group was coming from GSH. M4 is a di-GSH conjugate, and the sulfhydryl and amino groups were acquired from the respective GSH molecules. Unlike the formation of M1-M4, for the generation M5 and M6, the sulfhydryl came from NAC, and the amino group was acquired from NAL. The in vitro findings for M1-M6 provided important evidence for the formation of cis-enedial intermediate in microsomal reactions. M1, M2, M3, M5, and M6, which were found in microsomal incubations, were also detected in the urine samples of mice given LIM (Figure 3); M1, M2, M3, and M5 were detected in LIM treated mouse fecal samples as well (Figure 3). It appears that the primary LIM-derived GSH, $\mathrm{NAC}$, and/or NAL conjugates are excreted mainly through the urine with relatively trace amount they excreted through feces. However, M4, which identified as a LIM-derived diGSH conjugate, was not detected from the mouse urine and fecal samples after LIM treatment. Nevertheless, the observation of the GSH or NAC and NAL conjugates in urine and feces infers the metabolism of LIM to the cis-enedial intermediate in vivo. In addition, bioactivation studies with individual recombinant enzymes demonstrated that $\mathrm{P} 450$ $2 \mathrm{C} 9,2 \mathrm{C} 19$, and $3 \mathrm{~A} 4$ are enzymes responsible for the bioactivation of LIM (Figure 4(a)), with P450 3A4 as the most potent one. These findings facilitate our ongoing investigation of the biochemical mechanisms of LIM-induced enzyme inactivation.

In summary, the metabolic generation of the cis-enedial intermediate from LIM was evident in both in vitro and in vivo systems. The condensation reaction of the electrophilic intermediate with GSH or NAC and NAL gave six GSH or NAC and NAL conjugates derived from the 
cis-enedial intermediate of LIM. P450 3A4 was identified as the dominant participant in the catalysis leading to the formation of the reactive cis-enedial intermediate. The metabolite identification work performed herein enables us to better understand the mechanisms of LIM-induced enzyme inactivation processes.

\section{Conflicts of Interest}

The author(s) declared no potential conflicts of interest with respect to the research, authorship, and/or publication of this article.

\section{Authors' Contributions}

Yujie Deng and Yudong Fu contributed equally to this study.

\section{Acknowledgments}

The authors gratefully acknowledge the financial support by National Natural Science Foundation of China (81600684) and Natural Science Foundation of Shandong Province (ZR2016HQ16) to Yujie Deng.

\section{Supplementary Materials}

Supplementary 1. Figure 1: extract ion $(\mathrm{m} / \mathrm{z} 758 \rightarrow 683)$ chromatograms obtained from LC-LTQ MS analysis of microsomal incubations containing LIM, GSH, NAL, and $\mathrm{NADPH}$ in the absence microsomes (A), or in presence of HLMs (B) or MLMs (C). (D) Extracted ion $(\mathrm{m} / z$ 802 $\rightarrow 758)$ chromatogram obtained from LC-LTQ MS analysis of synthetic M2 and M2'. (E) MS/MS spectrum of M2 generated in microsomal incubations (M2' showed the same MS/MS spectrum). (F) MS/MS spectrum of synthetic M2 (synthetic M2' showed the same MS/MS spectrum).

Supplementary 2. Figure 2: extract ion $(\mathrm{m} / z 760 \rightarrow 685)$ chromatograms obtained from LC-LTQ MS analysis of microsomal incubations containing LIM, GSH, NAL, and NADPH in the absence microsomes (A), or in presence of HLMs (B) or MLMs (C). (D) Extracted ion $(\mathrm{m} / z 760 \rightarrow 685)$ chromatogram obtained from LC-LTQ MS analysis of synthetic M3 and M3'. (E) MS/MS spectrum of M3 generated in microsomal incubations (M3' showed the same MS/MS spectrum). (F) MS/MS spectrum of synthetic M3 (synthetic M3' showed the same MS/MS spectrum).

Supplementary 3. Figure 3: extract ion $(\mathrm{m} / \mathrm{z} 1065 \rightarrow 936)$ chromatograms obtained from LC-LTQ MS analysis of microsomal incubations containing LIM, GSH, NAL, and $\mathrm{NADPH}$ in the absence microsomes (A), or in presence of HLMs (B) or MLMs (C). (D) Extracted ion $(\mathrm{m} / z 1065 \rightarrow$ 936) chromatogram obtained from LC-LTQ MS analysis of synthetic M4 and M4'. (E) MS/MS spectrum of M3 generated in microsomal incubations $\left(\mathrm{M} 4^{\prime}\right.$ showed the same MS/MS spectrum). (F) MS/MS spectrum of synthetic M4 (synthetic M4' showed the same MS/MS spectrum).

Supplementary 4. Figure 4: extract ion $(\mathrm{m} / \mathrm{z} 632 \rightarrow 614)$ chromatograms obtained from LC-LTQ MS analysis of microsomal incubations containing LIM, NAC, NAL, and $\mathrm{NADPH}$ in the absence microsomes (A), or in presence of
HLMs (B) or MLMs (C). (D) Extracted ion $(\mathrm{m} / \mathrm{z} 632 \rightarrow$ 614) chromatogram obtained from LC-LTQ MS analysis of synthetic M6. (E) MS/MS spectrum of M6 generated in microsomal incubations. (F) MS/MS spectrum of synthetic M6.

\section{References}

[1] M. Lv, P. Xu, Y. Tian et al., "Medicinal uses, phytochemistry and pharmacology of the genus Dictamnus (Rutaceae)," Journal of Ethnopharmacology, vol. 171, no. 2, pp. 247-263, 2015.

[2] J. S. Jang, E. G. Seo, C. Han et al., "Four cases of toxic liver injury associated with Dictamnus dasycarpus," Korean Journal Hepatolpgy, vol. 14, no. 2, pp. 206-212, 2008.

[3] M. Sheehan and D. Atherton, "One-year follow up of children treated with Chinese medicinal herbs for atopic eczema," British Journal of Dermatology, vol. 130, no. 4, pp. 488-493, 1994.

[4] J. Sun, X. Wang, P. Wang, L. Li, W. Qu, and J. Liang, "Antimicrobial, antioxidant and cytotoxic properties of essential oil from Dictamnus angustifolius," Journal of Ethnopharmacology, vol. 159, no. 15, pp. 296-300, 2015.

[5] J. B. Sun, W. Qu, P. Wang, F. H. Wu, L. Y. Wang, and J. Y. Liang, "Degraded limonoids and quinoline alkaloids from Dictamnus angustifolius G. Don ex Sweet. and their antiplatelet aggregation activity," Fitoterapia, vol. 90, pp. 209-213, 2013.

[6] J. B. Sun, N. Jiang, M. Y. Lv et al., "Limonoids from the root bark of Dictamnus angustifolius: potent neuroprotective agents with biometal chelation and halting copper redox cycling properties," RSC Advances, vol. 5, no. 31, pp. 24750-24757, 2015.

[7] J. Sun, N. Jiang, M. Lv et al., "Anstifolines A and B, two dimeric furoquinoline alkaloids from the root bark of Dictamnus angustifolius," RSC Advances, vol. 6, no. 27, pp. 22550-22554, 2016.

[8] J. B. Sun, P. Wang, J. Y. Liang, and L. Chen, "Phytochemical and chemotaxonomic study on Dictamnus angustifolius G. Don ex Sweet (Rutaceae)," Biochemical Systematics and Ecology, vol. 68, pp. 74-76, 2016.

[9] Y. Jiang, S. Li, H. Chang, Y. Wang, and P. Tu, "Pressurized liquid extraction followed by high-performance liquid chromatography for determination of seven active compounds in Cortex Dictamni," Journal of Chromatography A, vol. 1108, no. 2, pp. 268-272, 2006.

[10] H. Iwata, Y. Tezuka, S. Kadota, A. Hiratsuka, and T. Watabe, "Mechanism-based inactivation of human liver microsomal CYP3A4 by rutaecarpine and limonin from Evodia fruit extract," Drug Metabolism and Pharmacokinetics, vol. 20, no. 1, pp. 34-45, 2005.

[11] Y. L. Han, H. L. Yu, D. Li et al., "Inhibitory effects of limonin on six human cytochrome P450 enzymes and P-glycoprotein in vitro," Toxicology in Vitro, vol. 25, no. 8, pp. 1828-1833, 2011.

[12] L. A. Peterson, "Reactive metabolites in the biotransformation of molecules containing a furan ring," Chemical Research in Toxicology, vol. 26, no. 1, pp. 6-25, 2012.

[13] D. P. Williams, D. J. Antoine, P. J. Butler et al., "The metabolism and toxicity of furosemide in the Wistar rat and CD-1 mouse: a chemical and biochemical definition of the toxicophore," Journal of Pharmacology and Experimental Therapeutics, vol. 322, no. 3, pp. 1208-1220, 2007.

[14] J. C. Erve, S. C. Vashishtha, W. DeMaio, and R. E. Talaat, "Metabolism of prazosin in rat, dog, and human liver microsomes 
and cryopreserved rat and human hepatocytes and characterization of metabolites by liquid chromatography/tandem mass spectrometry," Drug Metabolism and Disposition, vol. 35, no. 6, pp. 908-916, 2007.

[15] J. Loeper, V. Descatoire, P. Letteron et al., "Hepatotoxicity of germander in mice," Gastroenterology, vol. 106, no. 2, pp. 464-472, 1994.

[16] V. De Berardinis, C. Moulis, M. Maurice et al., "Human microsomal epoxide hydrolase is the target of germanderinduced autoantibodies on the surface of human hepatocytes," Molecular Pharmacology, vol. 58, no. 3, pp. 542-551, 2000.

[17] A. Druckova and L. J. Marnett, "Characterization of the amino acid adducts of the enedial derivative of teucrin A," Chemical Research in Toxicology, vol. 19, no. 10, pp. 1330-1340, 2006.

[18] D. Lin, K. Wang, X. Guo, H. Gao, Y. Peng, and J. Zheng, "Lysine-and cysteine-based protein adductions derived from toxic metabolites of 8-epidiosbulbin E acetate," Toxicology Letters, vol. 264, no. 15, pp. 20-28, 2016.

[19] D. Lin, X. Guo, H. Gao et al., "In vitro and in vivo studies of the metabolic activation of 8-epidiosbulbin E acetate," Chemical Research in Toxicology, vol. 28, no. 9, pp. 1737-1746, 2015.

[20] D. Lin, W. Li, Y. Peng et al., "Role of metabolic activation in 8epidiosbulbin E acetate-induced liver injury: mechanism of action of the hepatotoxic furanoid," Chemical Research in Toxicology, vol. 29, no. 3, pp. 359-366, 2016.

[21] D. Lin, C. Li, Y. Peng, H. Gao, and J. Zheng, "Cytochrome P450-mediated metabolic activation of diosbulbin B," Drug Metabolism and Disposition, vol. 42, no. 10, pp. 1727-1736, 2014.

[22] W. Li, D. Lin, H. Gao et al., "Metabolic activation of furan moiety makes Diosbulbin B hepatotoxic," Archives of Toxicology, vol. 90, no. 4, pp. 863-872, 2016. 

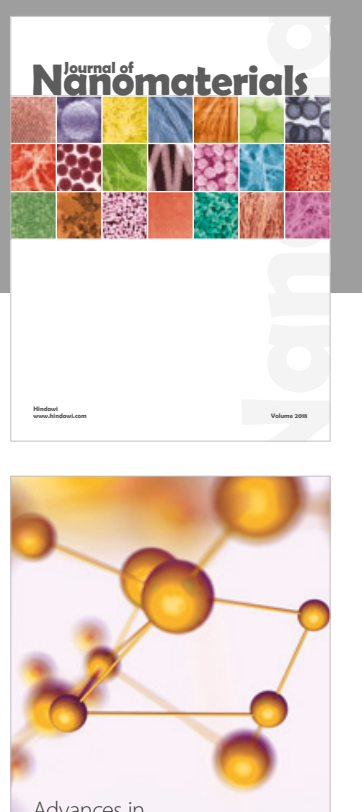

Physical Chemistry
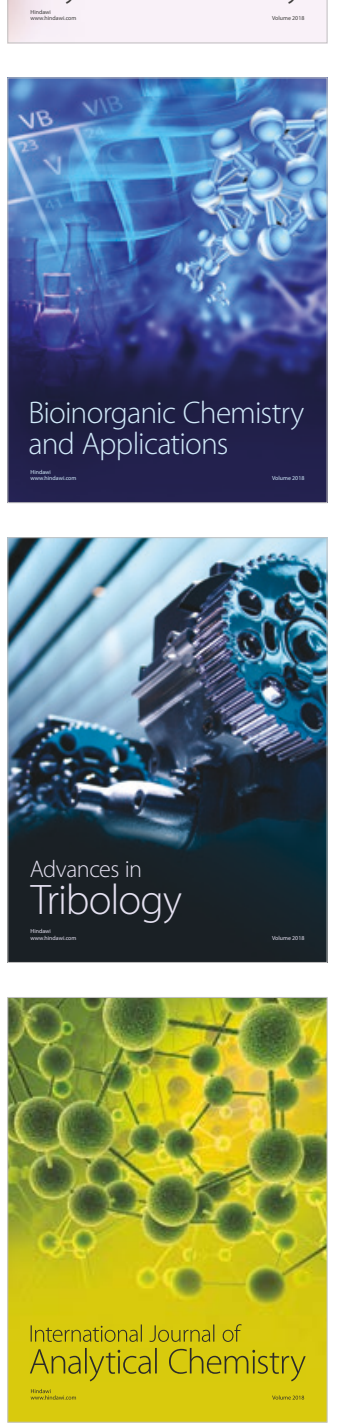

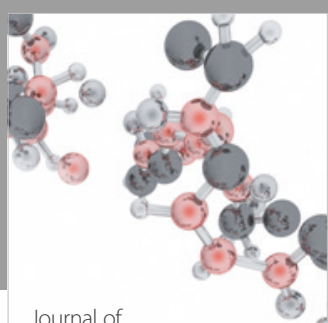

Analytical Methods

in Chemistry

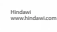

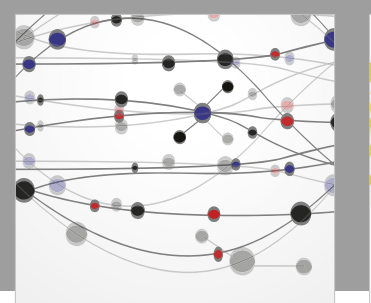

The Scientific World Journal

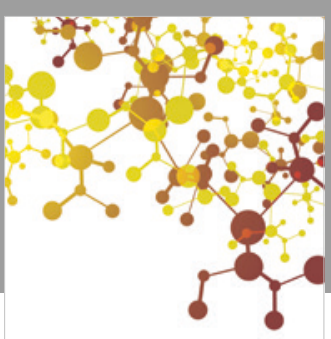

Journal of

Applied Chemistry
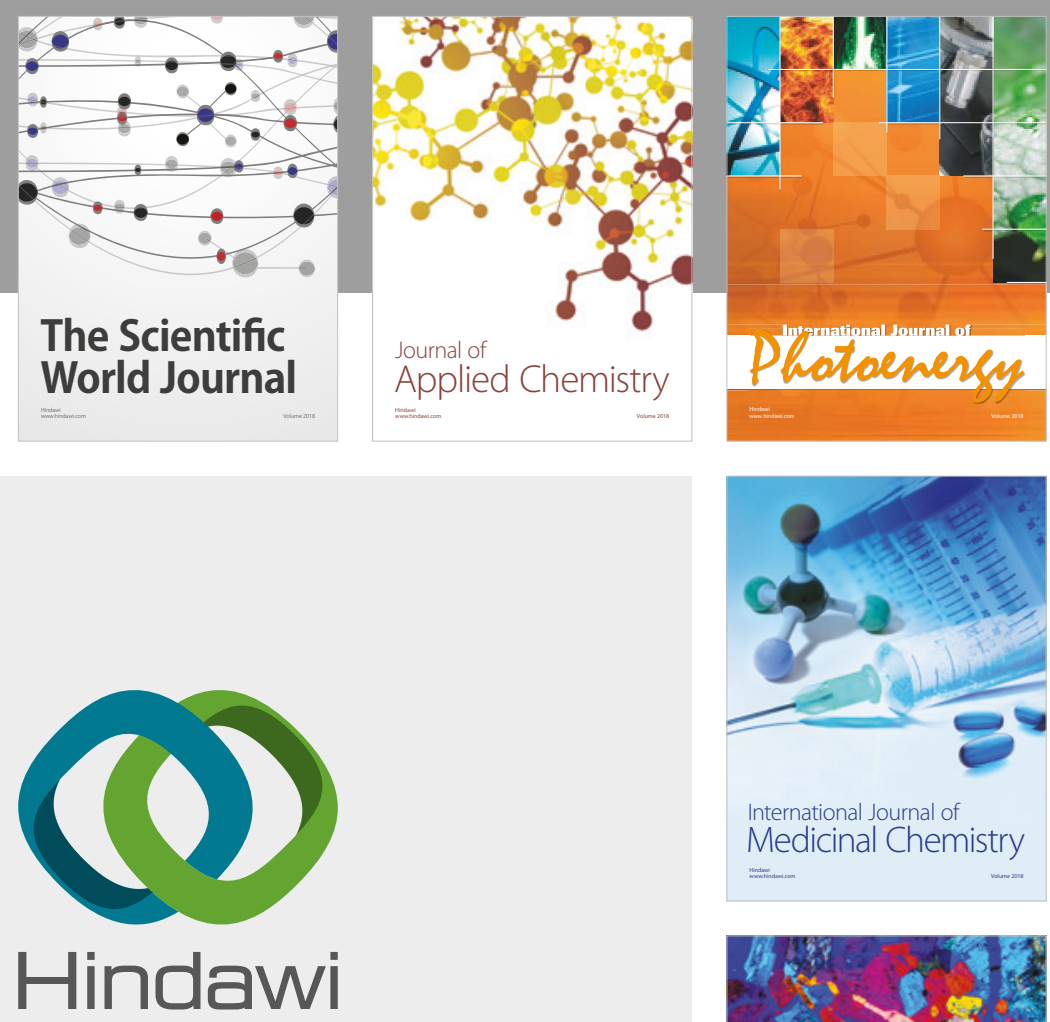

Submit your manuscripts at

www.hindawi.com
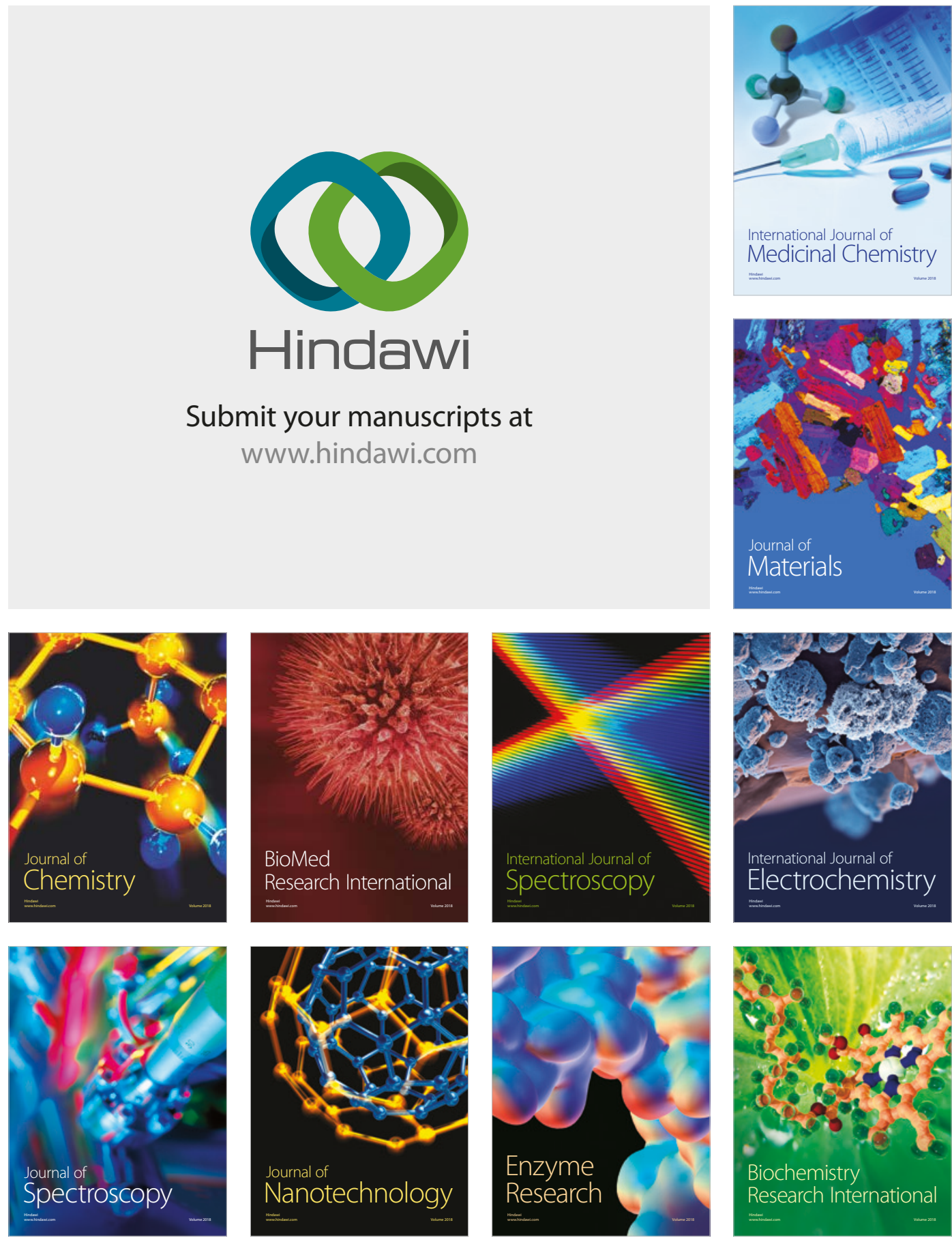
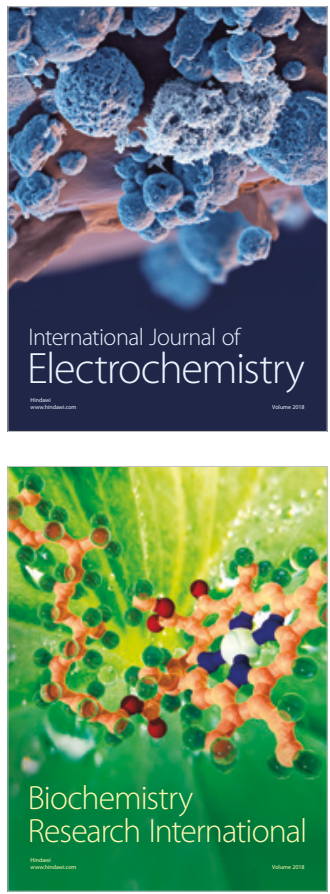